\title{
Validating Safecast data by comparisons to a U. S. Department of Energy Fukushima Prefecture aerial survey
}

\section{Abstract}

Safecast is a volunteered geographic information (VGI) project where the lay public uses hand-held sensors to collect radiation measurements that are then made freely available under the Creative Commons CC0 license. However, Safecast data fidelity is uncertain given the sensor kits are hand assembled with various levels of technical proficiency, and the sensors may not be properly deployed. Our objective was to validate Safecast data by comparing Safecast data with authoritative data collected by the U. S. Department of Energy (DOE) and the U. S. National Nuclear Security Administration (NNSA) gathered in the Fukushima Prefecture shortly after the Daiichi nuclear power plant catastrophe. We found that the two data sets were highly correlated, though the DOE/NNSA observations were generally higher than the Safecast measurements. We concluded that this high correlation alone makes Safecast a viable data source for detecting and monitoring radiation.

Keywords: Safecast, Volunteered Geographic Information, Fukushima Daiichi, Data Validation

\section{DISCLAIMER}

Oak Ridge National Laboratory is managed by UT-Batelle, LLC. This submission was written by one of the authors a acting in their own independent capacity and not on behalf of UT-Batelle, LLC, or its affiliates or successors.

\section{Introduction}

Volunteered Geographic Information (VGI) provides alternatives to government and corporate sponsored sources for determining the impact of natural or man-made disasters via crowd-sourced measurements (Goodchild, 2007). Ordinary citizens persons with smartphones or handheld sensors can make observations of disaster related phenomena that can supplement data gathered from traditional remotely sensed sources and ground-based equipment. However, sensing platforms are expensive to deploy, operate, and maintain, whereas VGI equipment is typically owned and operated by volunteers for comparatively little cost. Also, these citizen-based observations can cover areas from perspectives difficult to achieve with official sources, and with a very high spatial and temporal resolutions, especially in urban areas.

In fact, while space- and air-borne remote sensing can achieve a very high spatial resolution, in the order of a few centimeters in different parts of the electromagnetic spectrum, and vehicles can be deployed to capture data from the ground, satellites are not always overhead and are limited by atmospheric opacity (clouds and pollution), planes cannot remain airborne indefinitely, and ground vehicles have limited operating ranges and times.

Moreover, individuals intelligently evaluate their surroundings to focus their equipment on interesting scenes, whereas government or corporate managed sensors mechanically scan the environment without consideration to what is being observed, which means that these government and corporate sources may require more post-processing and analysis to mine useful information.

The Safecast VGI project uses "citizens as sensors" (Goodchild, 2007) to produce publicly available collection of radiation levels by time and location. Safecast participants collect these radiation measurements as a public service as well as for awareness of their own radiation exposure, and can be used as a citizen-led early warning system to detect radioactive leaks and hot spots. On March of 2015 there were over 27 million logged observations from around the globe. About $75 \%$ of the observations originated in Japan, primarily in Fukushima, surrounding prefectures, and in major Japanese cities. Up until 2013, virtually all measurements were confined to Japan. (Bonner and Brown, 2015).

Unfortunately VGI fidelity can be questionable because of possible operator reporting bias, poor data quality (such as the inclusion of generated test data), and equipment reliability and accuracy (Flanagin and Metzger, 2008). 
Though the Safecast organization has taken steps to ensure that the hardware is properly tested and calibrated before shipping (Safecast, 2015a), it is possible for the volunteer to make mistakes in assembling the sensor, particularly if they are inexperienced with putting together complicated electronic equipment. Moreover, the user may ignore equipment operating instructions (Safecast, 2015c), which may reduce observation quality. Though Safecast employs moderators to vet newly uploaded data (Brown et al., 2016), it is still theoretically possible for low quality data to be added to the publicly available database.

To address this open issue, we compared Safecast radiation observations with a similar set of observations made by the U. S. Department of Energy jointly with the U. S. National Nuclear Security Administration (DOE/NNSA) (Lyons and Colton, 2012). We found that the two datasets were strongly correlated, but that the DOE/NNSA observations were generally higher than the corresponding Safecast values. Later, we explore possible explanations for these differences. However, given the high correlation between the two datasets, we conclude that the Safecast data has utility for measuring environmental radiation.

\subsection{The 2011 Tohoku Earthquake and Tsunami}

On March 11, 2011 at 5:46:24UTC a 9.0 magnitude earthquake occurred $130 \mathrm{~km}$ east of Sendai, Honshu, Japan at a depth of approximately $30 \mathrm{~km}$ near the Pacific and North American plate subduction zone (U. S. Geological Survey, 2011). Earthquake models showed that the fault moved upwards by $30 \mathrm{~m}$ to $40 \mathrm{~m}$ over a $300 \mathrm{~km}$ by $150 \mathrm{~km}$ area with effects that were felt as far away as Korea, southeastern Russia, and China (U. S. Geological Survey, 2011). The plate shift was extreme enough to move the Earth's axis $25 \mathrm{~cm}$ and speed up its rotation by $1.8 \mu$ s per year (Chai, 2011). Moreover, the earthquake slid Honshu, the main island of Japan, $3.6 \mathrm{~m}$ to the east, while part of the Oshika Peninsula moved about $5.3 \mathrm{~m}$ towards the earthquake's epicenter (Norio et al., 2011).

The tsunami caused by this earthquake affected 20 different Pacific Rim countries with most of the damage occurring in Japan. It is estimated that the tsunami reached a peak of $38 \mathrm{~m}$ above mean sea level while penetrating up to $10 \mathrm{~km}$ inland (Norio et al., 2011). Over 300,000 buildings, 2,000 roads, and 50 bridges were damaged or destroyed. There were also approximately 15,000 casualties, 5,300 injured, and 4,600 missing people due to the tsnunami (U. S. Geological Survey, 2011). The combined earthquake and tsunami had an estimated initial overall economic impact of up to 183 billion US Dollars (Norio et al., 2011).

\subsection{The Fukushima Daiichi Nuclear Disaster}

The 11 nuclear power plants in northeastern Japan automatically shutdown when the earthquake struck (Norio et al., 2011). In spite of of these automatic safety procedures, the Fukushima Daiichi power plant suffered a level 7 catastrophic nuclear incident, the highest level on the International Nuclear and Radiological Event Scale (INES), due to earthquake and tsunami damage (Norio et al., 2011). The $5.7 \mathrm{~m}$ seawall at the Daiichi power plant was overcome by a $15 \mathrm{~m}$ high tsunami that flooded backup diesel generators and washed their fuel tanks into the ocean (Funabashi and Kitazawa, 2012), which meant that the power plant had no diesel generators to power the cooling systems (Nakamura and Kikuchi, 2011). In turn, this resulted in the partial meltdown of the reactor cores, which led to significant releases of radiation into the atmosphere and the ocean (Funabashi and Kitazawa, 2012; Nakamura and Kikuchi, 2011; Chino et al., 2011).

\subsection{The Advent of the Safecast Project}

Motivated by the lack of reliable and publicly available information regarding the ongoing Fukushima Daiichi nuclear power plant disaster, that same month a group of hobbyists organized the Safecast project, which focused on providing the means for citizens to collect and share radiation observations. The Safecast project logged their first observations with handbuilt radiation detectors in April, 2011, just one month after the tsunami struck Japan (Brown et al., 2016). The Safecast project is internationally crowdfunded and crowdsourced with over 650 handheld units and several stationary sensors that have contributed more than 27 million radiation measurements as of March 2015 (Bonner and Brown, 2015). In May 2014 there were over 14 million Safecast observations within Japan, though there were also millions of observations from Korea, Iraq, the United States, and other locations.

Figure 1 shows log-adjusted Safecast radiation observations for Japan, and depicts the radiation plume from the Fukushima Daiichi Nuclear Power Plant (FDNPP) spreading to the northwest about $50 \mathrm{~km}$ before turning and fading to the southwest. Most of the data follows the roadways because the hand held units are typically attached to car 
windows during observations, though there are also data gathered from ships off the east coast of the main island. The green circles indicate several permanent stationary sensors that also contribute observations to the Safecast database.

Safecast's current handheld radiation detector, the bGeigie Nano, is shown in Fig. 2, and is the fifth generation of their open source hardware design. It uses the LND 7317 radiation sensor, which is a $5.08 \mathrm{~cm}$ diameter pancake style radiation sensor that can detect alpha, beta, and gamma radiation using a Geiger-Müller tube filled with a mixture of neon and halogen gases (LND, Inc., 2011). The device also has a Global Positioning System (GPS) receiver to record the location of radiation readings. The Safecast detector records only the sensor output in counts per minute with time and location information and does not do any other manipulation of the saved data. For the display on the device, the counts per minute can be converted to either micro Sieverts per hour $(\mu \mathrm{Sv} / \mathrm{hr})$ or Becquerel per meter squared $\left(\mathrm{Bq} / \mathrm{m}^{2}\right)$, both based on ${ }^{137} \mathrm{Cs}$. Radiation observations are logged to a Secure Digital (SD) memory card, which can then be uploaded to the Safecast site; the data is freely available to the public via the Creative Commons CC0 license (Creative Commons, 2015). bGeigie Nano kits can be purchased online for roughly $\$ 450$, and then assembled by users within a few hours (Safecast, 2015b; Brown et al., 2016). The device is typically deployed by attaching it to the outside of a vehicle's window and driving through areas of interest, though users can also collect observations from their bGeigie Nano while walking (Brown et al., 2016).

\subsection{Safecast Data Validity}

The Safecast team has implemented quality control measures to ensure equipment accuracy. First, all the electronic components are factory tested before being shipped (Safecast, 2015a). Second, units are randomly selected, assembled, and undergo calibration tests at the Jülich Research Centre in Germany, QualTek in the US, and the International Atomic Energy Agency (IAEA) testing laboratory in Seibersdorf, Austria. The tested units have demonstrated $\pm 10 \%$ accuracy, which is the typical Safecast performance ( $\pm 15 \%$ is their maximum), which compares well to the normal industry calibration accuracy (Spinrad, 2011) and the recommended periodic check accuracy of $\pm 10 \%$ for medical uses (Zanzonico, 2008). Third, all data is checked by a team of domain experts before being accepted into the database; however, approximately $0.1 \%$ of uploads required such scrutiny (Brown et al., 2016).

The kits are built by volunteers of varying technical ability, which means that there may be assembly errors that may have a negative impact on accuracy. Moreover, though there are guidelines for using the bGeigie Nano units to mitigate data accuracy problems (Safecast, 2015c), there is no guarantee that the users have followed those guidelines when deploying their devices, which also can have an impact on the unit's accuracy.

Given these concerns, to date there has been no in depth analysis of the accuracy of the millions of Safecast radiation measurements. It is necessary to be confident that Safecast observations are reliable and accurate if they are to be used to supplement authoritative data for decision making. One means of checking Safecast data is to compare it with a set of observations made from different equipment that come from a trusted source, which is the strategy we have taken in this work. In the next section we describe the details of this approach.

\section{Material and Methods}

The Open Street Map (OSM) project is similar to Safecast in that it is a volunteer effort to freely provide global geospatial data. Individuals use GPSs, smartphones, or cameras to capture local spatial information that is then added to a central publicly accessible database. This database can then be used to create maps or to do route planning (Bennett, 2010). However, Volunteered Geographic Information (VGI) such as found with OSM can have problems with reliability, quality, and utility. That is, participants may be using faulty equipment or make observation errors that could negatively affect data quality (Flanagin and Metzger, 2008).

One way to validate VGI is to compare it with high fidelity data, such as from an authoritative and trusted source. OSM data for the London metropolitan area was compared to corresponding geospatial data from the British Ordnance Survey (BOS). London was selected because that is the first area OSM mapped, and so would have the oldest and therefore most reviewed data, as well as maximizing overlap between the two datasets (Haklay, 2010).

Safecast data is similar in nature to OSM's just by virtue of them both being VGI and, as such, just as there was an open question regarding OSM's quality since it was a form of VGI, the same question applied to Safecast. Therefore we took a similar approach to evaluating Safecast's observation fidelity by comparing Safecast observations with authoritative data. We chose to compare Safecast data with the DOE/NNSA aerial survey data gathered over the 
Fukushima Prefecture shortly after the nuclear disaster (Lyons and Colton, 2012) because these two datasets had significant overlapping spatiotemporal observations in that region.

\section{Experimental}

The DOE/NNSA dataset has 107,147 observations that cover roughly $20,000 \mathrm{~km}^{2}$ over the Fukushima Prefecture for a period of five weeks, from April 2nd through May 9th, 2011. Given that the observations were made several hundred meters above sea level, the data values were corrected to what they would be one meter above the ground presuming the ground or the air at this reference height is the reference. Also, since the observations were made on different days and the radiation from the elements ${ }^{134} \mathrm{Cs}$ and ${ }^{137} \mathrm{Cs}$ decay at different rates over time, to use that dataset you would have to take into consideration when the observations were made and which elements' radiation energy levels were measured to compensate for radioactive decay. Since this would be computationally cumbersome to do properly, the DOE/NNSA used the respective half lives of ${ }^{134} \mathrm{Cs}$ and ${ }^{137} \mathrm{Cs}$ to project forward all the observations to June 30, 2011 (Lyons and Colton, 2012).

The Safecast and DOE/NNSA data was compared by first clipping the Safecast data to the same geographical extent as the DOE/NNSA data, then considering only the Safecast observations for the same period - from April 2nd through June 30th - which resulted in 71,616 Safecast observations. Fig. 3 shows the distribution of observations between the two surveys for this period. We chose to emulate what was done for the DOE/NNSA aerial survey and extrapolated the remaining amount of Cs for the Safecast data to June 30, 2011, with the same assumption of a 1:1 ratio of the two Cs isotopes. The following formula was used to estimate the remaining amount of Cs radionuclides for June 30, 2011 (U. S. Occupational Safety \& Health Administration, 2015):

$$
A=A_{i} e^{-\left(0.693 t / T_{1 / 2}\right)}
$$

Where $A$ is the activity at some time of interest, $A_{i}$ is the activitity at the initial time, $t$ is the elapse time from the initial time to the time of interest, and $T_{1 / 2}$ is the given isotope's half life in the same units of time. The $T_{1 / 2}$ for ${ }^{134} \mathrm{Cs}$ is 2.06 years and ${ }^{137} \mathrm{Cs}$ is 30.17 years.

The DOE/NNSA used $\mathrm{mR} / \mathrm{hr}$ (miliRem per hour) as a unit of measure whereas Safecast used counts per minute (cpm), or the ionization events that the Geiger-Müller tube detects. The Safecast measurements were converted to use $\mathrm{mR} / \mathrm{hr}$ to facilitate comparison to the DOE/NNSA data using the following formula (Kozhuharov, 2014; Mallins, 2014; Dolezal, 2014):

$$
1 m R / h r=\frac{1}{3340} \mathrm{cpm}
$$

Note that we did not make additional adjustments for the devices measuring different energy levels. The constant in Eq. 2 includes this conversion.

Now that the two datasets were for the same area and time period, and also used the same units for measuring radiation, the next step was to do the comparison between them. However, the two datasets had distinctly dissimilar spatial characteristics, which posed a challenge for doing a direct comparison. For example, Fig. 4 shows that the DOE/NNSA areal survey covered large swathes of territory in a gridlike pattern; by contrast, Fig. 1a shows that the bulk of the Safecast observations were made along roads.

To compare the two datasets we chose to follow a similar approach taken by the Japanese Nuclear Regulation Authority (JNRA) for rasterizing radiation measurements. The JNRA uses the following steps to gather and share their radiation measurements (Japanese Nuclear Regulation Authority, 2014):

1. project the data to a 2D coordinate system, such as Universal Transverse Mercator (UTM)

2. overlay a polygonal grid onto the point data

3. average the measurements for each grid cell

4. assign that average to a corresponding cell in a separate raster image with the same dimensions

Following these steps to compare the DOE/NNSA to Safecast measurements, we first projected the two datasets to UTM Zone 54, then used a polygon grid overlay comprised of $500 \mathrm{~m}^{2}$ grid cells, and then averaged the radiation readings corresponding to each cell for both datasets. We then derived two raster images, one for the Safecast data, and 
the other for the DOE/NNSA, where each pixel value contained the corresponding average for each corresponding grid cell. We kept raster cells for which there were common set of observations to allow for direct comparisons between the two datasets. Fig. 5 shows the process of rasterizing and filtering grid cells for which there were observations from both the DOE/NNSA and Safecast.

\section{Results}

Fig. 6 shows the respective distributions of radiation observations between the DOE/NNSA aerial survey and Safecast measurements. Both datasets are similarly distributed with $\approx 80 \%$ of the observations being below $0.1 \mathrm{mR} / \mathrm{hr}$. However, the DOE/NNSA observations are slightly higher in value than the Safecast, and there are two DOE/NNSA readings that are much larger than all other observations.

Fig. 7 shows the respective spatial distributions of the DOE/NNSA and Safecast radiation measurements. There the two datasets appear visually to be highly correlated, which is supported by the pairwise $\operatorname{cor}\left(\overrightarrow{v_{d}}, \overrightarrow{v_{s}}\right)$ of 0.962 , where $\overrightarrow{v_{d}}$ corresponds to the vector of DOE/NNSA observations, and $\overrightarrow{v_{s}}$ the Safecast. However, the non-peak DOE/NNSA values appear to be higher than the corresponding Safecast observations.

Table 1: Summary statistics for coefficients for linear model of DOE/NNSA vs. Safecast coordinates shows correspondingly low p-values and standard errors.

\begin{tabular}{rrrrr}
\hline & Estimate & Std. Error & $\mathrm{t}$ value & $\operatorname{Pr}(>|\mathrm{t}|)$ \\
\hline (Intercept) & -0.0007 & 0.0010 & -0.73 & 0.4633 \\
DOE & 0.7418 & 0.0069 & 107.33 & 0.0000 \\
\hline
\end{tabular}

When the values of two identical sets of observations are plotted against each other, they normally align along the 45 degrees diagonal. However, when the sets are not identical but just very similar, as when they are off by a constant, it is still possible to observe a strong linear relationship. Fig. 8a shows a scatter plot of Safecast measurements against the corresponding DOE/NNSA with the regression line with 95\% confidence interval shown. The corresponding linear model has a p-value of less than 0.0001 , and an adjusted $R^{2}$ of 0.9262 . Table 1 shows the correspondingly low p-values and standard errors for the coefficients obtained through our statistical testing.

Results show that the DOE coefficient is less than one, providing supporting evidence that the DOE/NNSA values are generally higher with respect to Safecast values. This was saw earlier in Fig. 3. A Wilcoxon rank sum pairwise statistical test between the two datasets also supports the claim that the generally the DOE/NNSA data have higher values than much of the corresponding Safecast values $(\mathrm{p}<0.0001)$. In other words, Safecast generally underestimates the radiation levels.

\section{Discussion}

While the Safecast and DOE/NNSA data were strongly correlated, the DOE/NNSA radiation measurements were generally higher. This may have several possible explanations. First, though we applied the same DOE/NNSA extrapolation procedure of projecting Safecast data to June 30th, 2011, the bulk of the Safecast measurements were made later than the DOE/NNSA, as shown in Fig. 3, and so extant potassium and iodine radionuclides would not have been as prevalent as for the DOE/NNSA to be detected by the bGeigie Nano units; moreover, any precipitation made before or during the Safecast observations would remove some of the water soluble Cs. Second, the observed differences could also be because the Safecast and DOE/NNSA observations were made by different sensor technology. That is, Safecast used a Geiger-Müller tube containing neon and halogen and the DOE and NNSA deployed thallium activated sodium iodide crystal-based detectors. Thirdly, another contributing factor to the higher DOE/NNSA readings could be that the Safecast measurements were predominately made from automobiles while the DOE/NNSA used a C-12 fixed wing aircraft, which meant that the DOE/NNSA measurements had to be extrapolated to one meter above the ground, which was particularly challenging since the altitude had to be estimated given that aircraft's altimeter readings were inaccessible (Lyons and Colton, 2012), thus introducing a source of uncertainty. Fourth, given the strong linear relationship between the two datasets, another possibility is that the conversion factor that was used in Eq. 2 could be improved. 


\subsection{Compensating for Influence of Early Extant Radioactive Potassium and Iodine}

If the existence of higher amounts of radioactive potassium and iodine were contributing factors in making the DOE observations greater than for Safecast, then we should observe that the largest values dominate the earliest measurements. Indeed, Fig. 9 shows that the highest recorded DOE radiation observations were made on the second day of flights.

Given that, the two datasets may be significantly closer in value if we drop the first two days of DOE observations. However, then the concern would be that the spatial distribution between the two datasets may change such that a fair comparison is no longer possible, but this concern may be mitigated if the areas corresponding to the dropped observations were later re-measured. The left sub-figure in Fig. 10 shows the subset of the DOE observations that are within $500 \mathrm{~m}$ of Safecast data that corresponds to the first two days - or days of highest recorded measurements; the right sub-figure shows all the remaining DOE measures, also within $500 \mathrm{~m}$ of Safecast data. This shows that we can drop the first two days of DOE measurements with little impact on the spatial representation of data since the earliest observed areas were measured again by the DOE.

Table 2: Summary statistics for coefficients for linear model of DOE/NNSA vs. Safecast coordinates shows correspondingly low p-values and standard errors.

\begin{tabular}{rrrrr}
\hline & Estimate & Std. Error & $\mathrm{t}$ value & $\operatorname{Pr}(>|\mathrm{t}|)$ \\
\hline (Intercept) & -0.0007 & 0.0010 & -0.70 & 0.4846 \\
DOE & 0.7550 & 0.0070 & 107.79 & 0.0000 \\
\hline
\end{tabular}

Figure 11 shows the regression between the DOE observations with those first two days of measurements removed compared once more to the corresponding Safecast data, and which looks almost identical to the linear regression depicted in Fig. 8a. Table 2 corroborates this comparison in that it shows that the regression did not change much, though the adjusted $R^{2}$ did improve from 0.9262 to 0.9329 . However, a pairwise Wilcoxon rank sum test shows that, overall, the DOE measurements are still statistically higher than the corresponding Safecast observations $(p<0.0001)$. Therefore, we conclude that removing the earliest, higher DOE measurements had little overall impact on the differences between the two datasets, and so one or more of the other possible explanations posed earlier may be the cause.

\subsection{Summary}

Regardless of the higher DOE/NNSA radiation values, even after removing the first two days of DOE observations containing the highest values, the two datasets are still strongly correlated. They both described the same relative regions of high vs. low areas of radioactive contamination. In this regard, Safecast has shown that it can be used to detect radioactivity, such as in scenarios for improving disaster response to radiation producing events.

\section{Conclusions}

Volunteers for the Safecast project use handheld sensors to gather radiation measurements that are later freely shared with the public. Unfortunately, the fidelity of this volunteer gathered data may be questionable given that it relies on participants of varying levels of training and from equipment that they assembled themselves. With this in mind, we validated Safecast data by comparing it to U. S. Department of Energy's National Nuclear Security Administration observations made within the Fukushima Prefecture shortly after the March 2001 Daiichi nuclear power plant disaster occurred. We found that the two sets of observations were highly correlated, but that the DOE/NNSA measurements were somewhat higher. Nonetheless, despite the differences, we feel that the Safecast data is useful for public safety given that it identified similar regions of high radiation as did the DOE/NNSA.

One possible cause of the DOE/NNSA and Safecast data differences included significant periods of nonoverlapping observations. One way to address that problem would be to perform similar types of comparisons as was used in this work between Safecast data and other authoritative datasets with observations made in the same span. Given that the Japanese government continues to regularly monitor radiation levels via aerial surveys of the Fukushima Prefecture, and likewise Safecast measurements continue to be made in that same area, then similar studies can be made between those datasets. Likewise, other comparisons could be made where other authoritative data sufficiently overlaps with Safecast data. 


\section{7. Acknowledgements}

This research was funded by ONR grants N00014-13-1-0784 and N00014-14-1-0208. We would also like to thank ${ }_{257}$ Kalin Kozhuharov, Nick Dolezal, and Alex Mallins for their help with the Safecast cpm to $\mathrm{mR} / \mathrm{hr}$ unit conversions, ${ }_{258}$ and other participants on the Safecast mailing list for their feedback. We would also like to thank the reviewers for 259 their valuable feedback. 


\section{References}

Bennett, J., 2010. OpenStreetMap. Packt Publishing Ltd, Olton Birmingham, GBR, iSBN: 978-1-84719-750-4.

Bonner, S., Brown, A., March 2015. The Safecast Report.

URL http: //blog. safecast .org/2015/03/the-safecast-report/

Brown, A., Franken, P., Bonner, S., Dolezal, N., Moross, J., Jun. 2016. Safecast: successful citizen-science for radiation measurement and communication after Fukushima. Journal of Radiological Protection 36 (2), S82-S101.

URL http: //stacks.iop.org/0952-4746/36/i=2/a=S82?key=crossref . 191ea617bad97e08b87e3a7ad72e6781

Chai, C., March 2011. Japan's quake shifts earth's axis by 25 centimetres.

URL http: //www. webcitation.org/5x95t0CLU

Chino, M., Nakayama, H., Nagai, H., Terada, H., Katata, G., Yamazawa, H., 2011. Preliminary estimation of release amounts of 131i and 137cs accidentally discharged from the fukushima daiichi nuclear power plant into the atmosphere. Journal of nuclear science and technology 48 (7), 1129-1134.

Creative Commons, December 2015. Cc0 1.0 universal (cc0 1.0) public domain dedication.

URL http://creativecommons .org/publicdomain/zero/1.0/

Dolezal, N., August 2014. Converting Safecast measurements to mR/h. Personal communication.

Flanagin, A. J., Metzger, M. J., 2008. The credibility of volunteered geographic information. GeoJournal 72, 137-148.

Funabashi, Y., Kitazawa, K., 2012. Fukushima in review: A complex disaster, a disasterous response. Bulletin of the Atomic Scientists 68 (2), 9-21.

Goodchild, M., 2007. Citizens as sensors: The world of volunteered geography. GeoJournal 69 (4), 211-221.

Haklay, M., July 2010. How good is volunteered geographical information? A comparative study of OpenStreetMap and Ordnance Survey datasets. Environment and Planning B: Planning and Design 37 (4), 682173.

URL http://ideas.repec.org/a/pio/envirb/v37y2010i4p682-703.html

Japanese Nuclear Regulation Authority, November 2014. Monitoring information of environmental radioactivity level. http://radioactivity.nsr.go.jp/en/.

URL http://radioactivity.nsr.go.jp/en/

Kozhuharov, K., August 2014. Converting Safecast measurements to mR/h. Personal communication

LND, Inc., 2011. LND 7317 Technical Specification.

URL http: //www. Indinc. com/products/17/

Lyons, C., Colton, D., 2012. Aerial Measuring System in Japan. Health Physics 102 (5), 509 - 515.

URL http://ovidsp.tx.ovid.com/sp-3.13.1a/ovidweb.cgi?QS2=434f4e1a73d37e8ce55b227ffaae5fa18cd3c69d19578a71a527e854f 162 eac138

Mallins, A., August 2014. Converting Safecast measurements to $\mathrm{mR} / \mathrm{h}$. Personal communication.

Nakamura, A., Kikuchi, M., 2011. What we know, and what we have not yet learned: Triple disasters and the fukushima nuclear fiasco in japan. Public Administration Review 71 (6), 893-899.

URL http://dx.doi.org/10.1111/j.1540-6210.2011.02437.x

Norio, O., Ye, T., Kajitani, Y., Shi, P., Tatano, H., 2011. The 2011 eastern japan great earthquake disaster: Overview and comments. International Journal of Disaster Risk Science 2 (1), 34-42. URL http://dx.doi.org/10.1007/s13753-011-0004-9

Safecast, December 2015a. About calibration and the bgeigie nano. URL http://blog.safecast.org/faq/about-calibration-and-the-bgeigie-nano/

Safecast, December 2015b. bgeigie nano kit. URL http: //blog. safecast .org/bgeigie-nano/

Safecast, December 2015c. Nano Operation Manual.

URL https ://github.com/Safecast/bGeigieNanoKit/wiki/Nano-Operation-Manual

Safecast, August 2015d. Safecast Maps. URL http://blog. safecast.org/maps/

Spinrad, P., 2011. John iovine: Geiger counter sanity check. Makezine. URL http://makezine.com/2011/04/25/john-iovine-geiger-counter-sanity-check/

U. S. Geological Survey, March 2011. Magnitude 9.0 - NEAR THE EAST COAST OF HONSHU, JAPAN. URL http: //earthquake.usgs.gov/earthquakes/eqinthenews/2011/usc0001xgp/

U. S. Occupational Safety \& Health Administration, December 2015. Ionizing Radiation Equations. URL https://www.osha.gov/SLTC/radiationionizing/introtoionizing/ionizingradequations.html

Zanzonico, P., 2008. Routine quality control of clinical nuclear medicine instrumentation: a brief review. Journal of Nuclear Medicine (49). 


\section{List of Figures}

1 These are screenshots from the Safecast web site of log adjusted radiation observations in $\mu \mathrm{Sv} / \mathrm{hr}$ for all of Japan and the vicinity near the FDNPP. Individual observations are overlaid on linearly interpolated values. Note that most observations follow along roadways, though the green circles represent permanent, stationary radiation sensors that also contribute to the Safecast network. The radiation plume from the Daiichi nuclear power plant can be seen following a northwesterly path for about $50 \mathrm{~km}$ before turning and fading southwest. (Safecast, 2015d) . . . . . . . . . . . . . .

2 These images show the front and back of the bGeigie Nano, and is the current handheld unit for contributing radiation observations to the Safecast database. This is the fifth generation of the bGeigie handheld sensor, which was released in 2013. (bGeigie Nano images courtesy of PSU's Geoinformatics and Earth Observation Laboratory) . . . . . . . . . . . . . . . . . . . .

3 This illustrates the number of samples gathered by day during the DOE/NNSA aerial survey of the Fukushima Prefecture from April 2nd through May 9th, 2011, and the Safecast observations made from April 24th, through June 30th, 2011. This shows that the bulk of the DOE/NNSA observations were made earlier in the same period than Safecast. However, both datasets had their respective observations extrapolated forward to June 30,2011 for easy comparison. . . . . . . . . . . . . .

4 DOE/NNSA radiation observations made over the Fukushima Prefecture from April 2nd through May

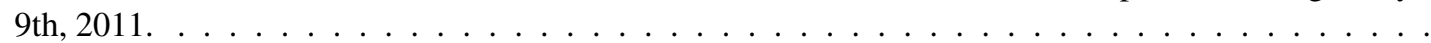

5 The DOE/NNSA and Safecast datasets were point observations that were translated into a corresponding set of raster images to make comparisons easier. A grid of $500 \mathrm{~m}^{2}$ cells was overlaid over the common area for the DOE/NNSA and Safecast observations as shown in the inset diagrams. A raster image for each set of observations was generated from the average of the respective observations for each grid cell, and grid cells for which there were no observations for both datasets were discarded. The location of the Fukushima Daiichi nuclear power plant is shown at the radiation hazard symbol along with $10 \mathrm{~km}$ concentric rings from the power plant shown up to $30 \mathrm{~km}$. . . . . . .

6 This is a histogram of radiation intensities for DOE/NNSA and Safecast log adjusted observations in units of $\mathrm{mR} / \mathrm{h}$ and with a bin size of 0.01 . The number, or count, of observations for the corresponding bin values are shown, and indicates that the two sets of observations have similar distributions. However, the DOE/NNSA measurements are slightly larger than the corresponding Safecast observations as shown by their respective medians, with the gold line representing the DOE/NNSA median, and the blue dotted line for the Safecast. There are also two outliers of DOE/NNSA observations that are much higher than other measurements that are highlighted in the box on the right of the figure.

7 These level plots depict the distribution of log adjusted radiation observations between the two datasets. The distributions appear to be highly correlated, though the DOE/NNSA non-peak observations are higher than the corresponding Safecast values, particularly for the areas of the highest level of measurements. . . . . . . . . . . . . . . . . . . . . .

8 Linear model of DOE/NNSA coordinates plotted against Safecast and a violin plot showing the respective measurement distributions. . . . . . . . . . . . . . . . . . . . .

9 From April 2nd through May 9th, 2011, the DOE/NNSA made 15 flights over the Fukushisma Prefecture to gather radiation observations, and this scatter plot aggregates those observations by each day. Note, some horizontal jitter and alpha transparency was applied to mitigate overplotting. The highest radiation observations were made on April 3, 2011. . . . . . . . . . . . . . .

10 Both figures depict the DOE radiation measurements made within $500 \mathrm{~m}$ of the corresponding Safecast observations. The left figure shows the 647 DOE observations made between April 2nd and 3rd, 2011, which also contained the highest measured values. The right shows the remaining 4,533 observations, which were made between April 4th and May 9th, 2011. This shows that dropping the first two days of DOE observations does not have a significant impact on the overall spatial coverage. . . . . . . . .

11 Linear regression between DOE and Safecast observations with the first two days of DOE observations, which contained the highest values, dropped. . . . . . . . . . . . . . . . 20 


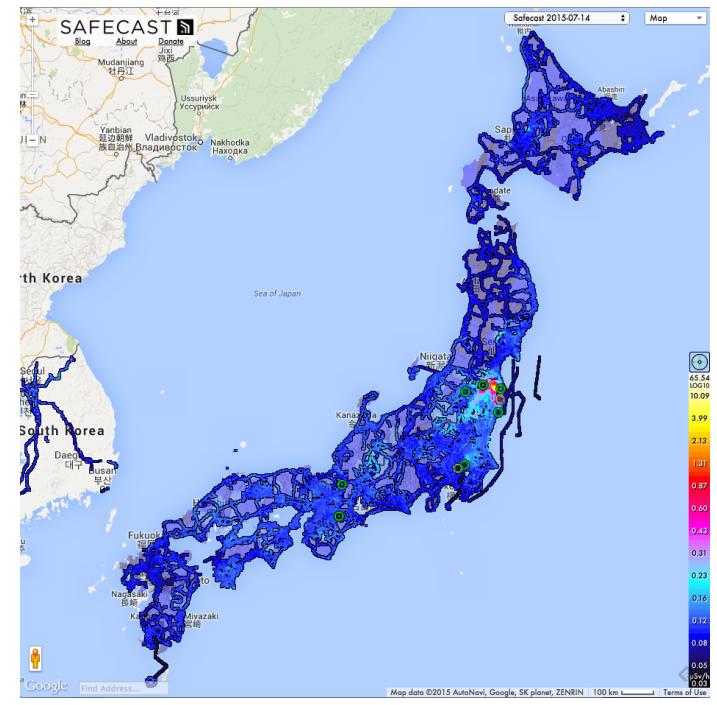

(a) Safecast observations for all of Japan.

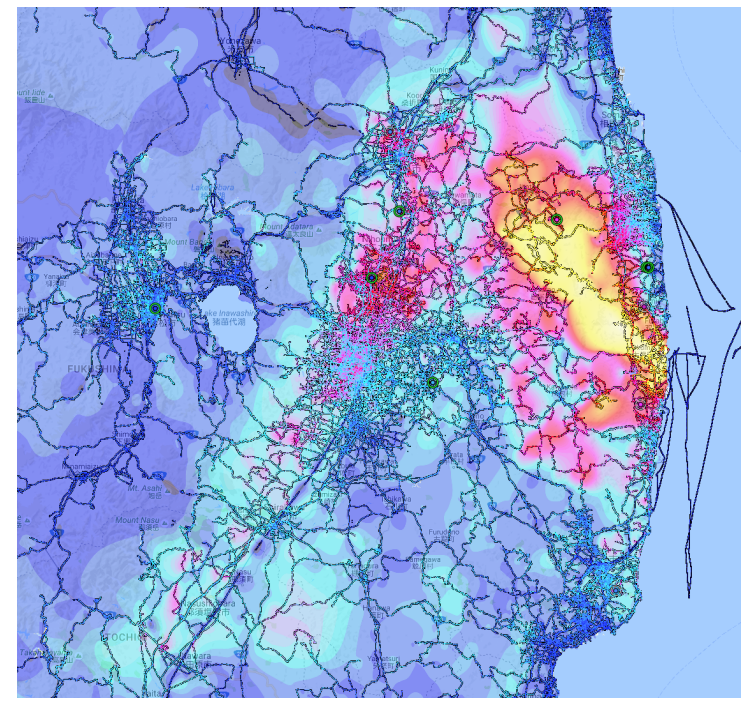

(b) Safecast observations near the Fukushima Daiichi nuclear power plant.

Figure 1: These are screenshots from the Safecast web site of log adjusted radiation observations in $\mu \mathrm{Sv} / \mathrm{hr}$ for all of Japan and the vicinity near the FDNPP. Individual observations are overlaid on linearly interpolated values. Note that most observations follow along roadways, though the green circles represent permanent, stationary radiation sensors that also contribute to the Safecast network. The radiation plume from the Daiichi nuclear power plant can be seen following a northwesterly path for about $50 \mathrm{~km}$ before turning and fading southwest. (Safecast, 2015d) 

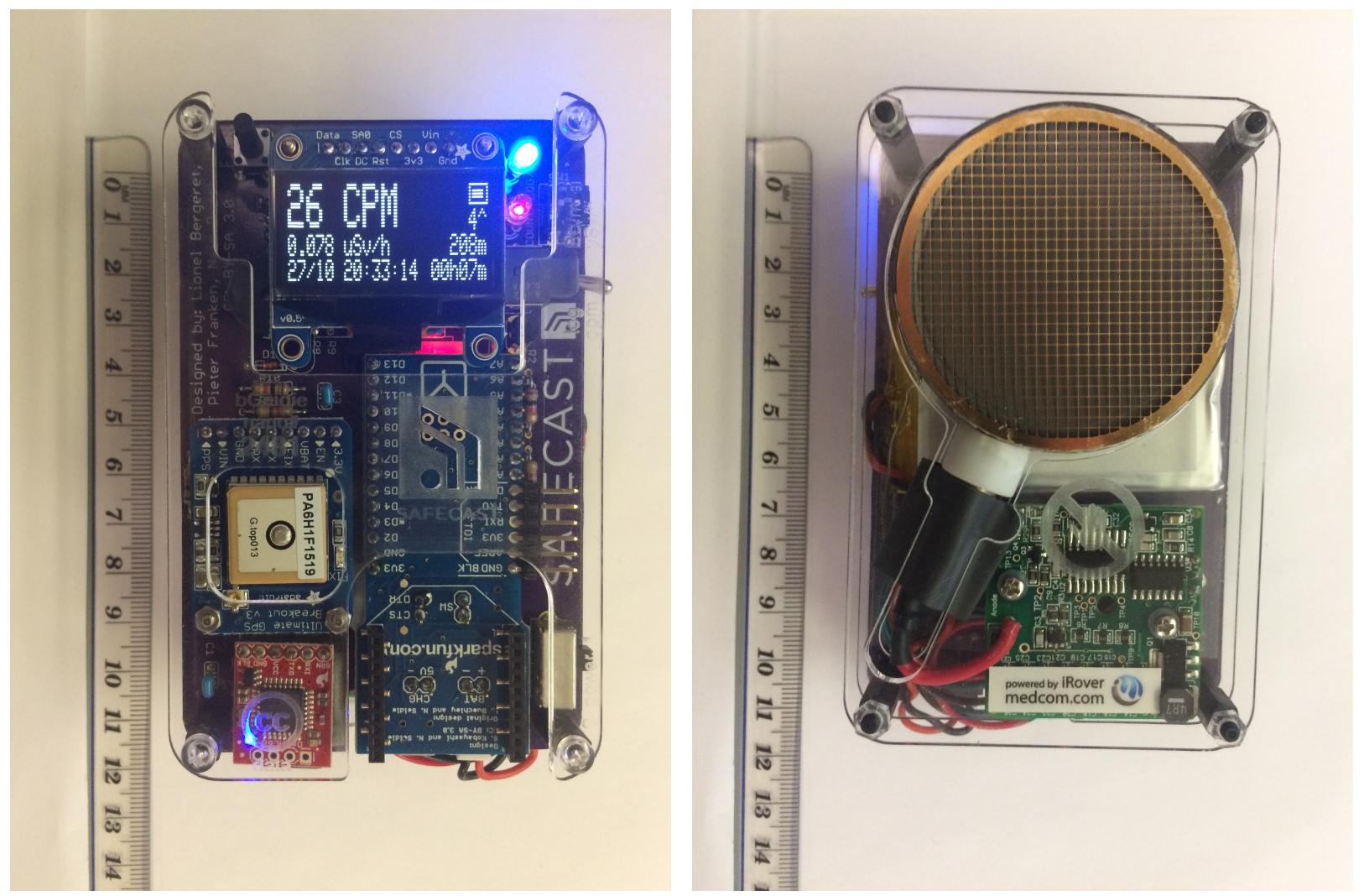

Figure 2: These images show the front and back of the bGeigie Nano, and is the current handheld unit for contributing radiation observations to the Safecast database. This is the fifth generation of the bGeigie handheld sensor, which was released in 2013. (bGeigie Nano images courtesy of PSU's Geoinformatics and Earth Observation Laboratory) 


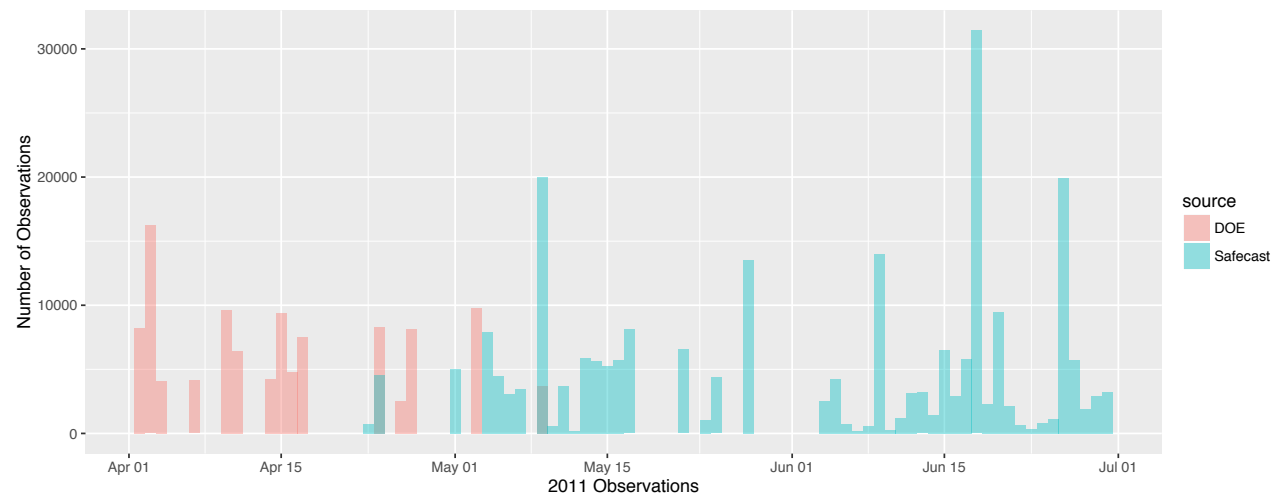

Figure 3: This illustrates the number of samples gathered by day during the DOE/NNSA aerial survey of the Fukushima Prefecture from April 2nd through May 9th, 2011, and the Safecast observations made from April 24th, through June 30th, 2011. This shows that the bulk of the DOE/NNSA observations were made earlier in the same period than Safecast. However, both datasets had their respective observations extrapolated forward to June 30, 2011 for easy comparison. 


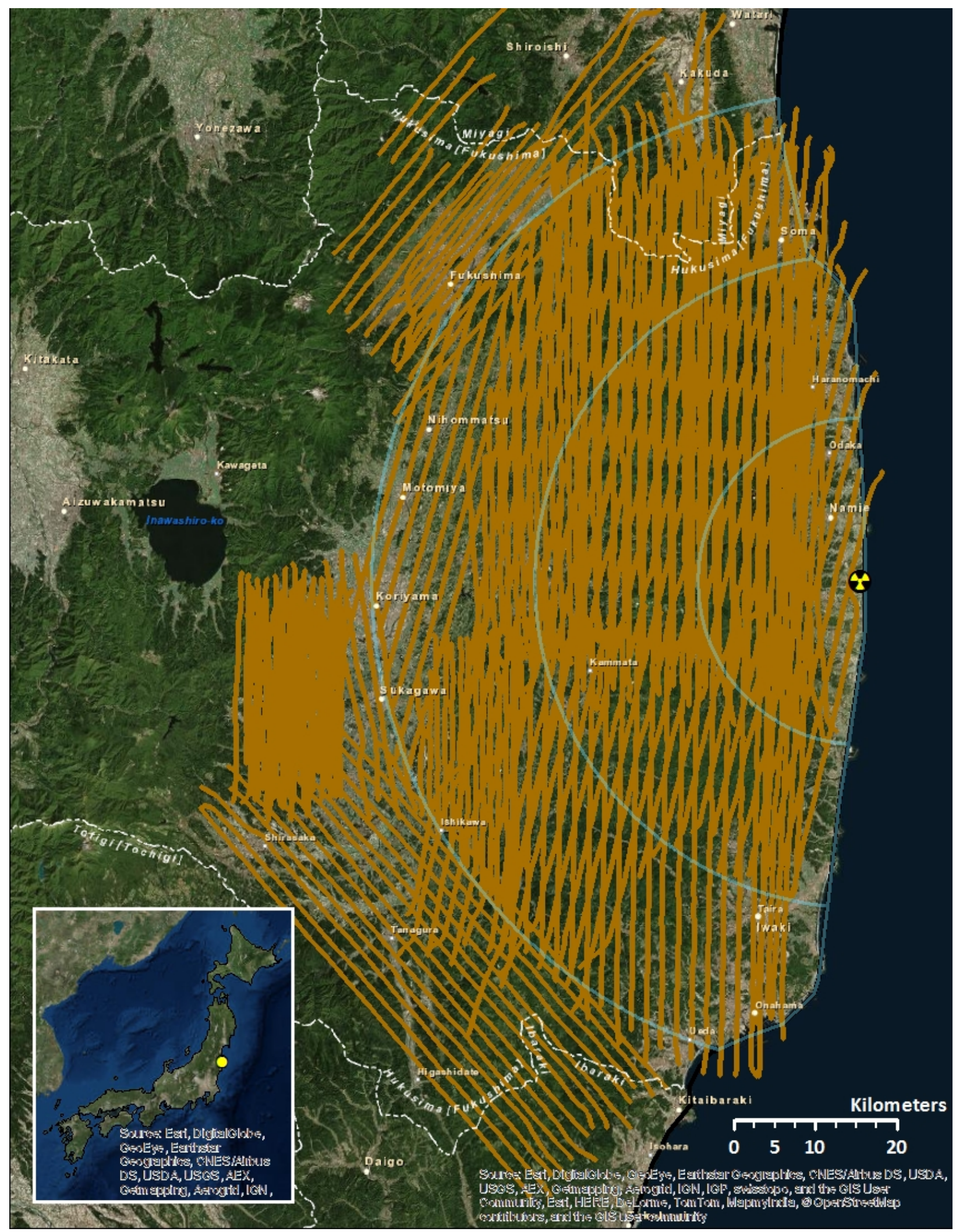

Figure 4: DOE/NNSA radiation observations made over the Fukushima Prefecture from April 2nd through May 9th, 2011. 


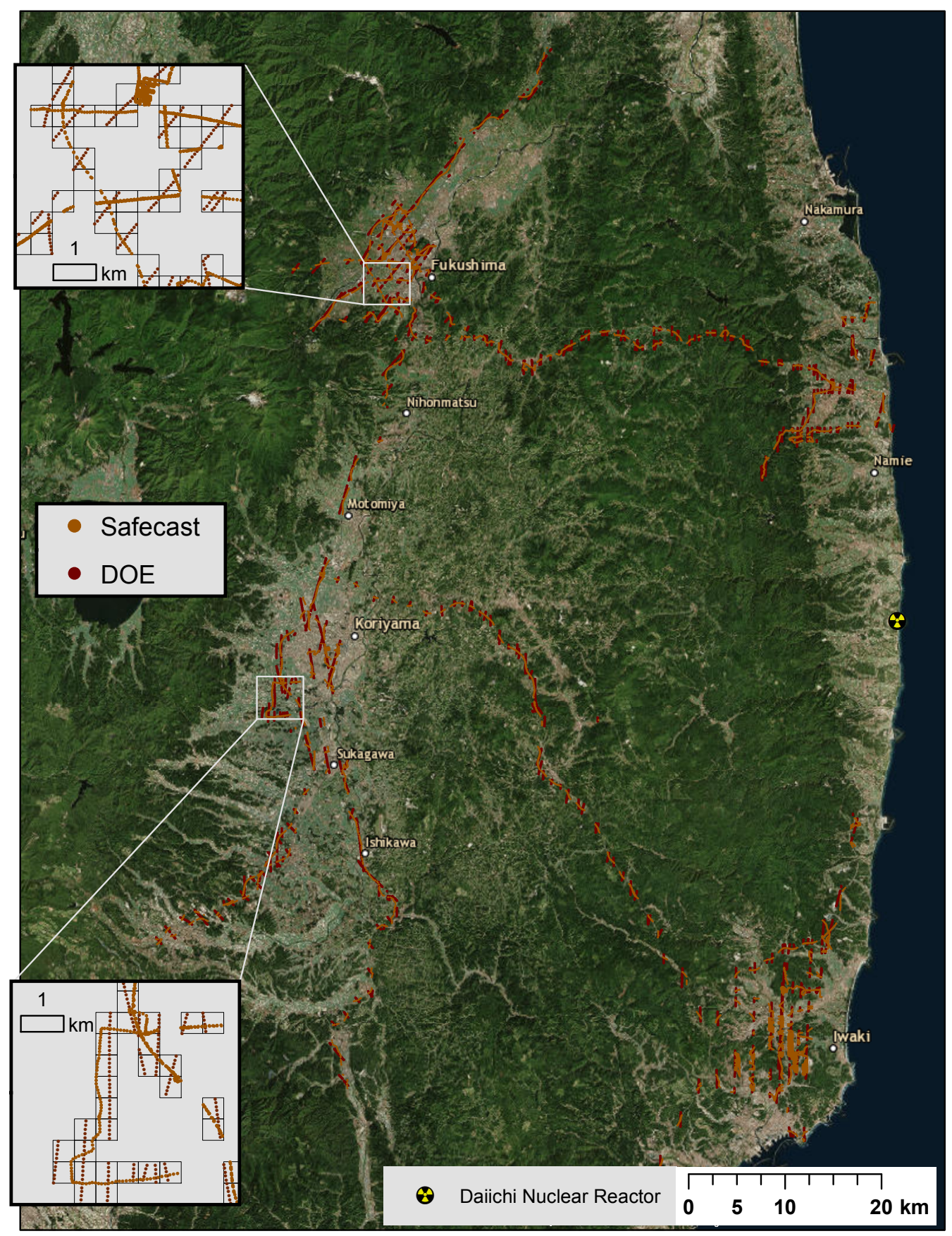

Figure 5: The DOE/NNSA and Safecast datasets were point observations that were translated into a corresponding set of raster images to make comparisons easier. A grid of $500 \mathrm{~m}^{2}$ cells was overlaid over the common area for the DOE/NNSA and Safecast observations as shown in the inset diagrams. A raster image for each set of observations was generated from the average of the respective observations for each grid cell, and grid cells for which there were no observations for both datasets were discarded. The location of the Fukushima Daiichi nuclear power plant is shown at the radiation hazard symbol along with $10 \mathrm{~km}$ concentric rings from the power plant shown up to $30 \mathrm{~km}$. 


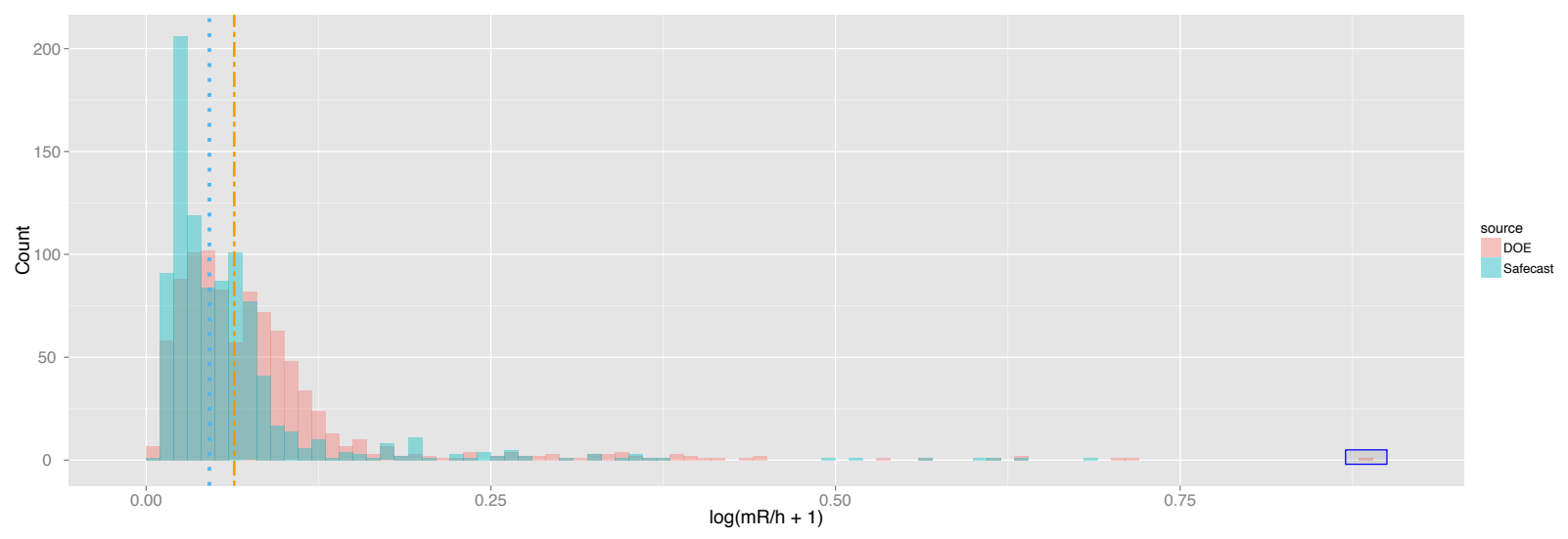

Figure 6: This is a histogram of radiation intensities for DOE/NNSA and Safecast log adjusted observations in units of $\mathrm{mR} / \mathrm{h}$ and with a bin size of 0.01. The number, or count, of observations for the corresponding bin values are shown, and indicates that the two sets of observations have similar distributions. However, the DOE/NNSA measurements are slightly larger than the corresponding Safecast observations as shown by their respective medians, with the gold line representing the DOE/NNSA median, and the blue dotted line for the Safecast. There are also two outliers of DOE/NNSA observations that are much higher than other measurements that are highlighted in the box on the right of the figure. 


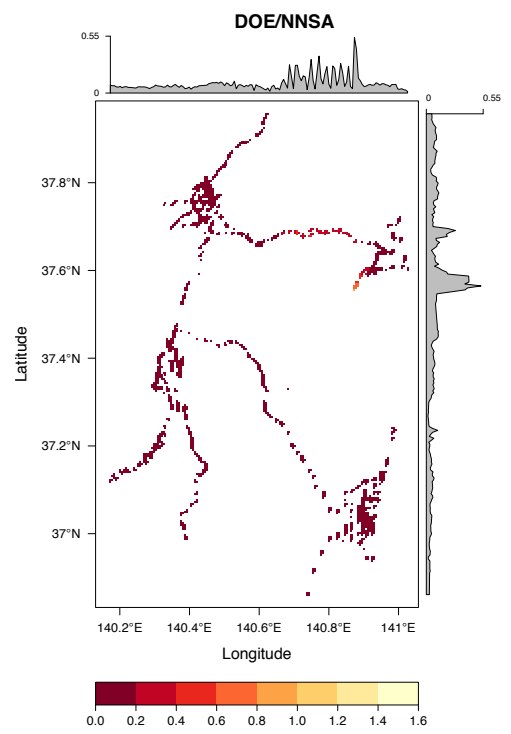

(a) Level plot of the distribution of DOE/NNSA observations.

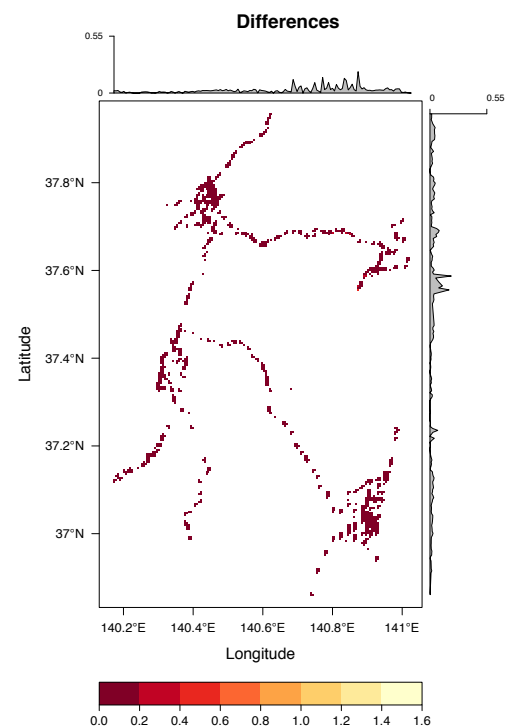

(b) Level plot of the differences be- (c) Level plot of the Safecast data

tween the DOE/NNSA and Safecast observations.

observations.

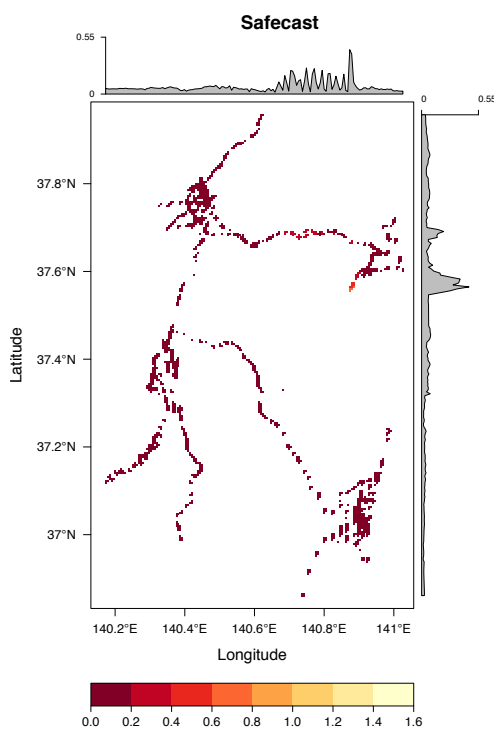

Figure 7: These level plots depict the distribution of log adjusted radiation observations between the two datasets. The distributions appear to be highly correlated, though the DOE/NNSA non-peak observations are higher than the corresponding Safecast values, particularly for the areas of the highest level of measurements. 


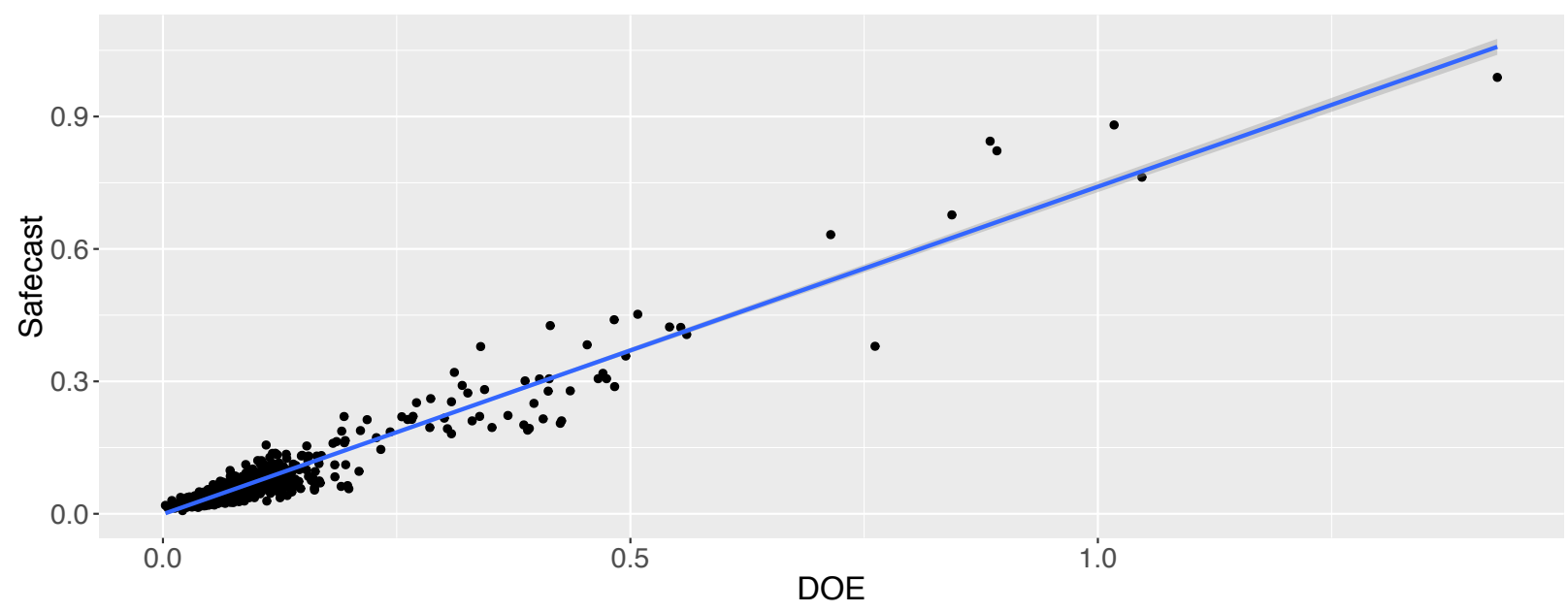

(a) Linear regression model of DOE/NNSA vs. Safecast observations in units of $\mathrm{mR} / \mathrm{hr}$ with linear regression $95 \%$ confidence interval region. That is, for every raster cell, the DOE/NNSA measurement is the x coordinate, and the Safeast the y. If the observations were identical, then the points would be in a $45^{\circ}$ line. That the slope is less than $45^{\circ}$ is another indicator that the DOE/NNSA values are somewhat higher.

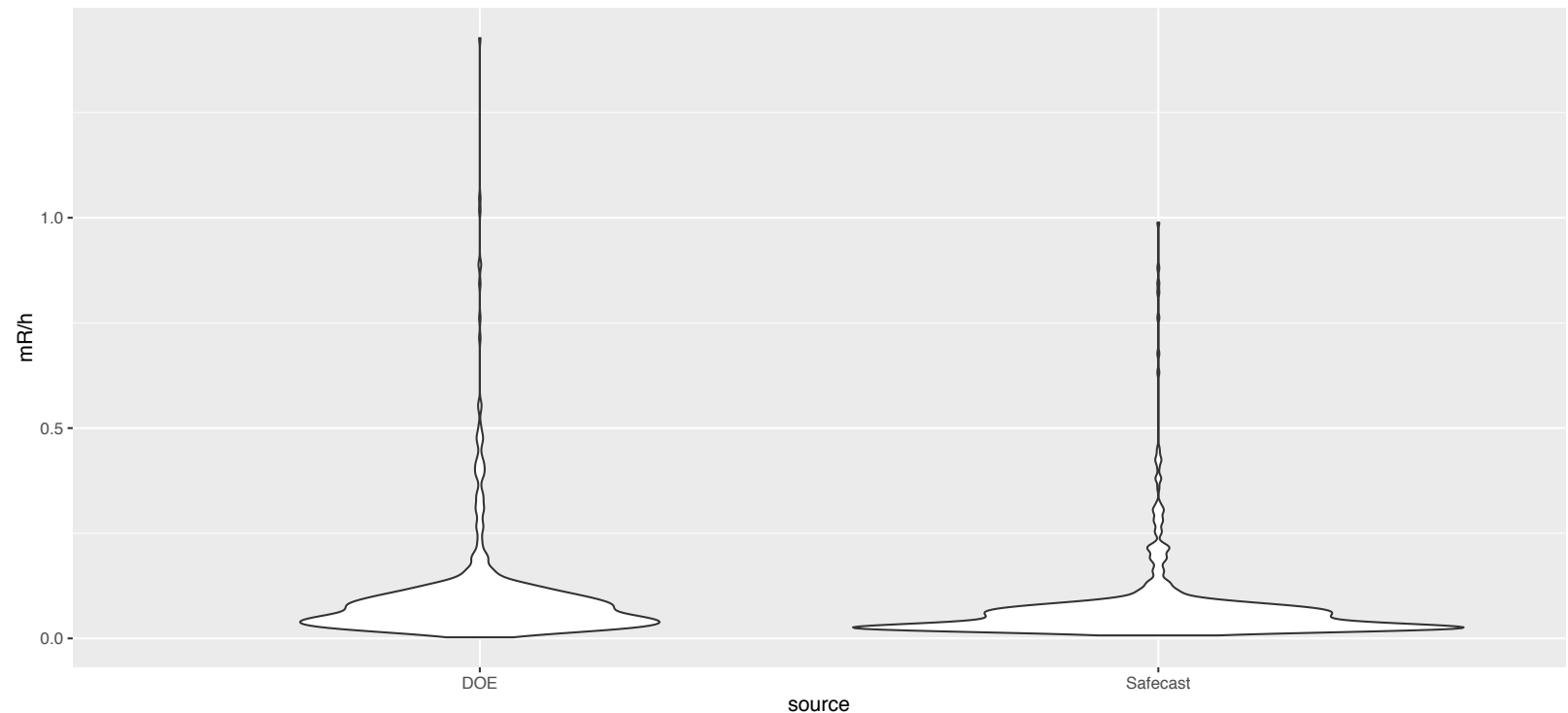

(b) Violin plot that shows the differences between the DOE/NNSA and Safecast measurement distributions, and shows that the distribution of the Safecast observation values tend to be lower than that of the DOE/NNSA.

Figure 8: Linear model of DOE/NNSA coordinates plotted against Safecast and a violin plot showing the respective measurement distributions. 


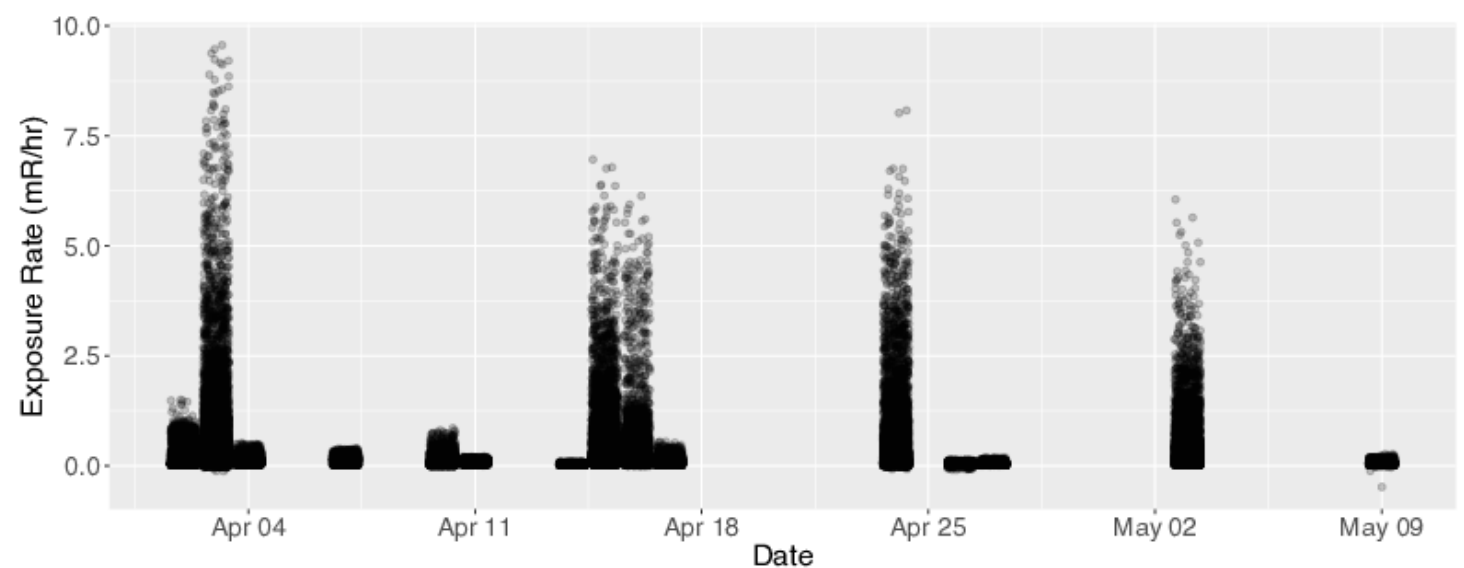

Figure 9: From April 2nd through May 9th, 2011, the DOE/NNSA made 15 flights over the Fukushisma Prefecture to gather radiation observations, and this scatter plot aggregates those observations by each day. Note, some horizontal jitter and alpha transparency was applied to mitigate overplotting. The highest radiation observations were made on April 3, 2011. 


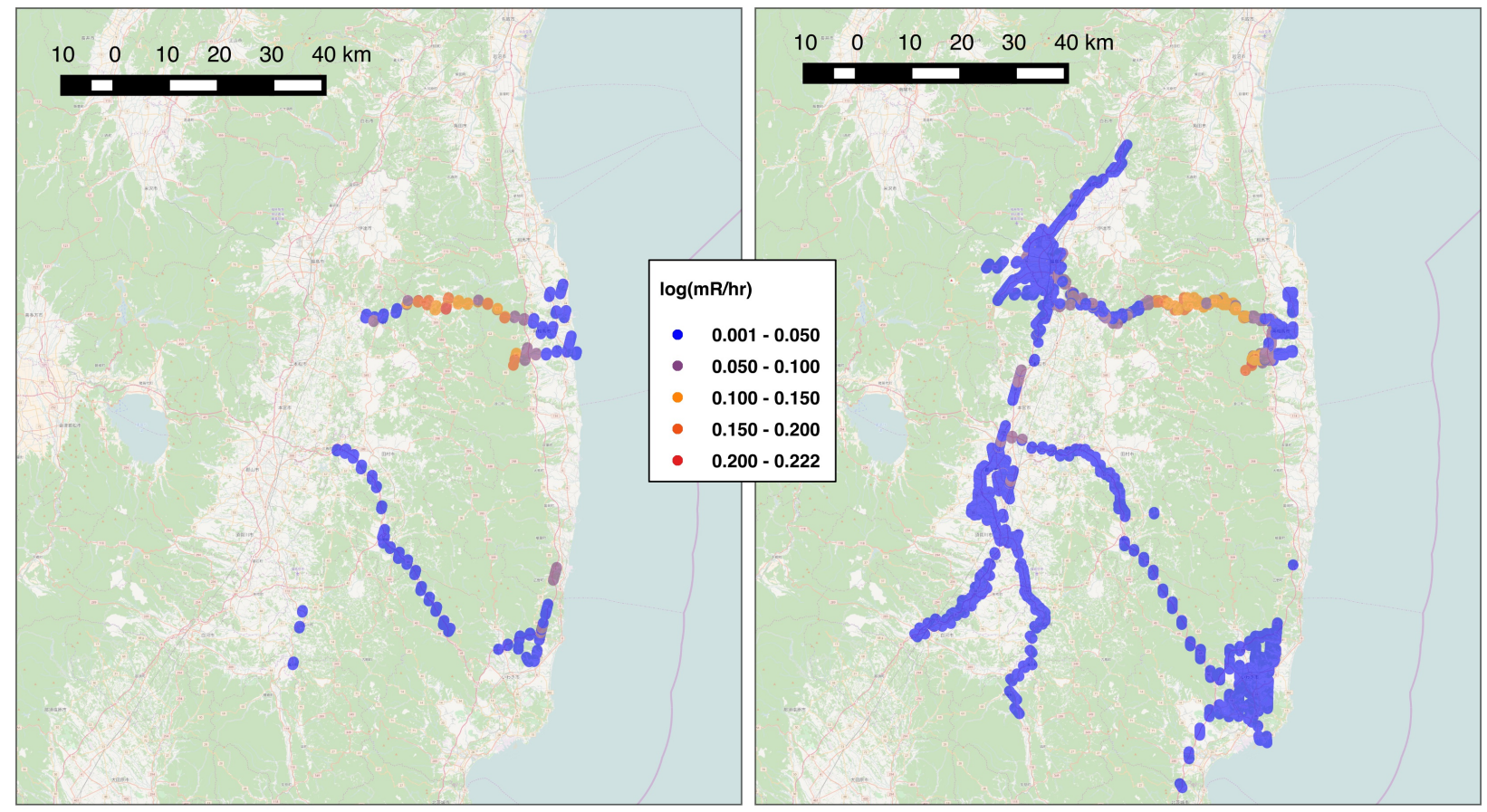

Figure 10: Both figures depict the DOE radiation measurements made within $500 \mathrm{~m}$ of the corresponding Safecast observations. The left figure shows the 647 DOE observations made between April 2nd and 3rd, 2011, which also contained the highest measured values. The right shows the remaining 4,533 observations, which were made between April 4th and May 9th, 2011. This shows that dropping the first two days of DOE observations does not have a significant impact on the overall spatial coverage. 


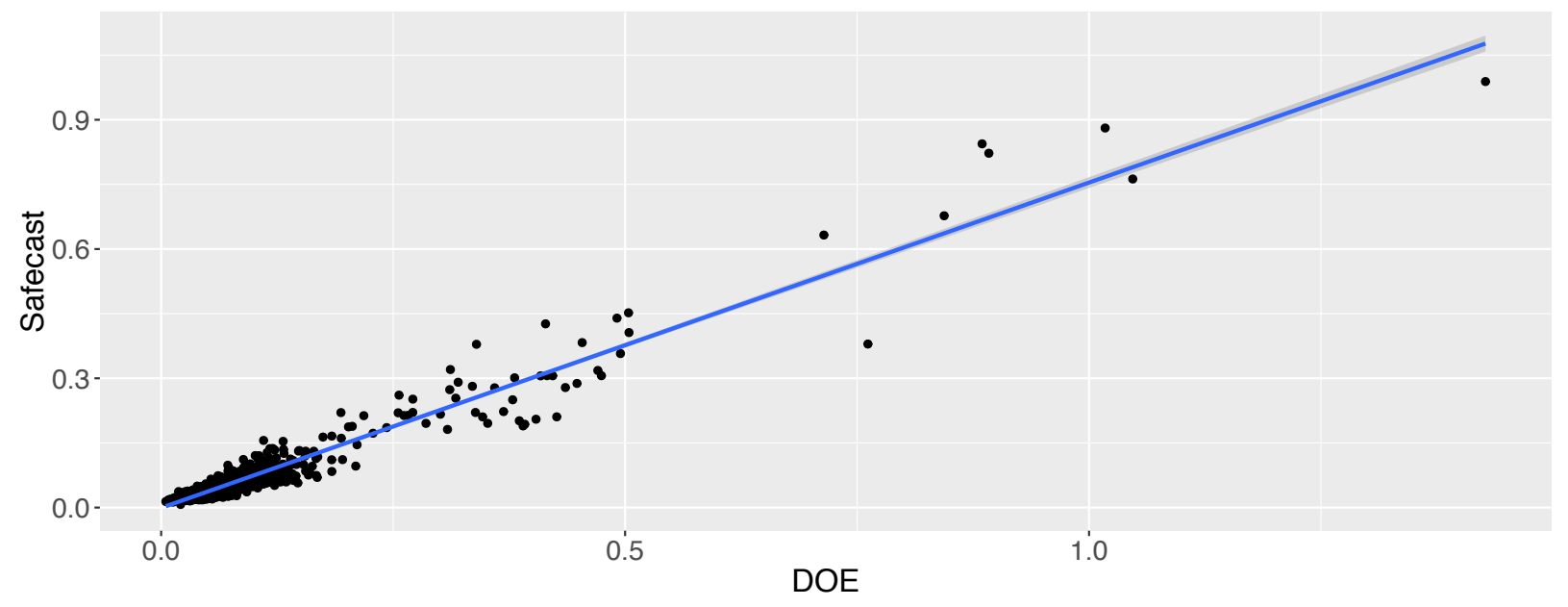

Figure 11: Linear regression between DOE and Safecast observations with the first two days of DOE observations, which contained the highest values, dropped. 